\title{
Circulating Tumor Cell Adhesion in Microcirculation
}

\author{
Lanlan Xiao", ${ }^{1,}$, Yang Liu ${ }^{1 *}$, Shuo $\mathrm{Chen}^{2}$, Bingmei $\mathrm{Fu}^{3}$ \\ ${ }^{1}$ Department of Mechanical Engineering, The Hong Kong Polytechnic University, \\ 11 Yuk Choi Rd, Hung Hom, Hong Kong \\ 14902368r@polyu.connect.hk; yang.liu@polyu.edu.hk \\ ${ }^{2}$ School of Aerospace Engineering and Applied Mechanics, Tongji University, \\ 1239, Siping Rd, Yangpu Dist, Shanghai, China \\ schen_tju@tongji.edu.cn \\ ${ }^{3}$ Department of Biomedical Engineering, The City College of the City University of New York \\ 160 Convent Ave, New York, NY, USA \\ fu@ccny.cuny.edu
}

\section{Extended Abstract}

Most cancer-related deaths are due to the metastasis. Blood microcirculation provides the principal pathway for delivering the circulating tumor cell (CTC) to distant organs to form secondary tumor. One of the hypotheses for initiating CTC extravasation is the attachment to the vascular endothelium through the formation of receptor-ligand bonds. The transition from initial attachment to firm adhesion largely depends on the blood flow conditions, which is lack of quantitative data. Experimental findings showed that CTC attachment mostly occurs at the venular part of the microcirculation [1], which implies that low flow rate facilitates CTC adhesion. As the white blood cell (WBC) has similar processes as CTC, most models developed for WBC are suitable for CTC. Existing studies indicated that leukocyte margination and adhesion are pronounced within an intermediate hematocrit range of $H t \approx 0.1-0.3[2,3]$ and the margination of CTC depends on its deformability [4] as well as its relative size to the vessel [5]. Furthermore, increasing the extent of RBC aggregation also increased the firm adhesion of WBCs to the endothelium [2, 6-8].

This study aims at studying the effects of haematocrit, vessel size, flow rate as well as RBC aggregation on CTC adhesion in idealized microvessels by a particle-based method - dissipative particle method. The membrane of cells was represented by a spring-based network and intercellular interaction between RBCs was modelled by a Morse potential function presented by Liu, et al. [9] based on depletion-mediated assumption. CTC adhesion was described by adhesive dynamics model developed by Hammer and Apte [10]. The CTC adhesion was found to be closely linked with its confinement. In the microvessel with a diameter of 5/3 of the CTC size, the CTC has a larger number of receptor-ligand bonds formed on the cell surface with the haematocrit due to a growing wall-directed force. But with the increase in the microvessel size, an enhanced lift force induced by the collision between RBCs and CTC at high haematocrit inhibits the CTC attachment. In addition, the velocity of CTC is larger than the average blood velocity in a smaller microvessel and the ratio of CTC velocity to the mean blood velocity drops to around 1 in a larger microvessel and even the CTC flows slower than the blood stream at $H t=0.1$ as the cell nearly moves near the vessel wall. Also, the presence of CTC increases the blood flow resistance, which is more pronounced for the case of CTC adhesion. Moreover, the strong tendency of RBCs migrating towards the vessel at a higher flow rate enables the detached CTC to contact with the vessel wall again. And the induced large deformation increases the number of receptor-ligand bonds for the adherent CTC. In the aggregating RBC suspensions, an enhanced CTC adhesion can be found because it enables the CTC to stably roll along the vessel wall at the low flow rate. And an additional wall-directed force provided by the RBC aggregates further compresses the CTC, leading to an increasing number of receptor-ligand bonds.

\section{Acknowledgement}

Supports given by HKRGC PolyU 5202/13E, PolyU G-YBG9, NSFC 51276130, and NIH SC1 CA153325-01 are gratefully acknowledged. 


\section{References}

[1] A. B. Al-Mehdi, K. Tozawa, A. B. Fisher, L. Shientag, A. Lee and R. J. Muschel, "Intravascular origin of metastasis from the proliferation of endothelium-attached tumor cells: a new model for metastasis," Nature Medicine, vol. 6, pp. 100-102, 2000.

[2] K. B. Abbitt and G. B. Nash, "Rheological properties of the blood influencing selectin-mediated adhesion of flowing leukocytes," American Journal of Physiology-Heart and Circulatory Physiology, vol. 285, pp. H229-H240, 2003.

[3] D. A. Fedosov and G. Gompper, "White blood cell margination in microcirculation," Soft Matter, vol. 10, pp. 29612970, 2014.

[4] M. R. King, K. G. Phillips, A. Mitrugno, T. R. Lee, A. M. de Guillebon, S. Chandrasekaran, et al., "A physical sciences network characterization of circulating tumor cell aggregate transport," Am J Physiol Cell Physiol, vol. 308, pp. C792-802, 2015.

[5] N. Takeishi, Y. Imai, T. Yamaguchi and T. Ishikawa, "Flow of a circulating tumor cell and red blood cells in microvessels," Physical Review E, vol. 92, 2015.

[6] C. Sun and L. L. Munn, "Influence of erythrocyte aggregation on leukocyte margination in postcapillary expansions: A lattice Boltzmann analysis," Physica A: Statistical Mechanics and its Applications, vol. 362, pp. 191-196, 2006.

[7] M. J. Pearson and H. H. Lipowsky, "Influence of erythrocyte aggregation on leukocyte margination in postcapillary venules of rat mesentery," American Journal of Physiology-Heart and Circulatory Physiology, vol. 279, pp. H1460H1471, 2000.

[8] L. L. Munn and M. M. Dupin, "Blood cell interactions and segregation in flow," Annals of Biomedical Engineering, vol. 36, pp. 534-544, 2008.

[9] Y. L. Liu, L. Zhang, X. D. Wang and W. K. Liu, "Coupling of Navier-Stokes equations with protein molecular dynamics and its application to hemodynamics," International Journal for Numerical Methods in Fluids, vol. 46, pp. 1237-1252, 2004.

[10] D. A. Hammer and S. M. Apte, "Simulation of Cell Rolling and Adhesion on Surfaces in Shear-Flow - General Results and Analysis of Selectin-Mediated Neutrophil Adhesion," Biophysical Journal, vol. 63, pp. 35-57, 1992. 